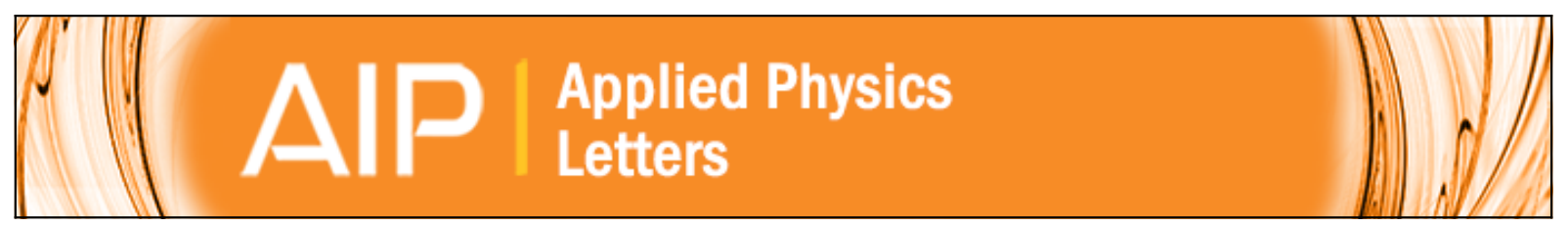

\title{
Ultrafast dynamics of type-II GaSb/GaAs quantum dots
}

K. Komolibus, T. Piwonski, K. Gradkowski, C. J. Reyner, B. Liang, G. Huyet, D. L. Huffaker, and J. Houlihan

Citation: Applied Physics Letters 106, 031106 (2015); doi: 10.1063/1.4906106

View online: http://dx.doi.org/10.1063/1.4906106

View Table of Contents: http://scitation.aip.org/content/aip/journal/apl/106/3?ver=pdfcov

Published by the AIP Publishing

\section{Articles you may be interested in}

Thermal emission in type-II GaSb/GaAs quantum dots and prospects for intermediate band solar energy conversion

J. Appl. Phys. 111, 074514 (2012); 10.1063/1.3703467

Time-resolved amplified spontaneous emission in quantum dots

Appl. Phys. Lett. 97, 251106 (2010); 10.1063/1.3529447

Room-temperature operation type-II GaSb/GaAs quantum-dot infrared light-emitting diode

Appl. Phys. Lett. 96, 123503 (2010); 10.1063/1.3371803

Complex emission dynamics of type-II GaSb/GaAs quantum dots

Appl. Phys. Lett. 95, 061102 (2009); 10.1063/1.3202419

Ultrafast dynamics of InAs/GaAs quantum-dot microdisk lasers

Appl. Phys. Lett. 78, 3397 (2001); 10.1063/1.1376437

High-Voltage Amplifiers

- Voltage Range from $\pm 50 \mathrm{~V}$ to $\pm 60 \mathrm{kV}$

- Current to $25 \mathrm{~A}$

Electrostatic Voltmeters

- Contacting \& Non-contacting

- Sensitive to $1 \mathrm{mV}$

- Measure to $20 \mathrm{kV}$
ENABLING RESEARCH AND

INNOVATION IN DIELECTRICS,

ELECTROSTATICS, MATERIALS, PLASMAS AND PIEZOS

TRek www.trekinc.com 


\title{
Ultrafast dynamics of type-II GaSb/GaAs quantum dots
}

\author{
K. Komolibus, ${ }^{1,2}$ T. Piwonski, ${ }^{1,2}$ K. Gradkowski, ${ }^{1,2}$ C. J. Reyner, ${ }^{3}$ B. Liang, ${ }^{3}$ G. Huyet,${ }^{1,2,4}$ \\ D. L. Huffaker, ${ }^{3}$ and J. Houlihan ${ }^{5}$ \\ ${ }^{1}$ Centre for Advanced Photonics and Process Analysis, Cork Institute of Technology, Cork, Ireland \\ ${ }^{2}$ Tyndall National Institute, University College Cork, Lee Maltings, Cork, Ireland \\ ${ }^{3}$ Department of Electrical Engineering and California NanoSystems Institute, University of California - Los \\ Angeles, Los Angeles, California 90095, USA \\ ${ }^{4}$ National Research University of Information Technologies, Mechanics and Optics, Saint Petersburg, Russia \\ ${ }^{5}$ School of Science, Waterford Institute of Technology, Waterford, Ireland
}

(Received 18 November 2014; accepted 6 January 2015; published online 20 January 2015)

\begin{abstract}
In this paper, room temperature two-colour pump-probe spectroscopy is employed to study ultrafast carrier dynamics in type-II GaSb/GaAs quantum dots. Our results demonstrate a strong dependency of carrier capture/escape processes on applied reverse bias voltage, probing wavelength and number of injected carriers. The extracted timescales as a function of both forward and reverse bias may provide important information for the design of efficient solar cells and quantum dot memories based on this material. The first few picoseconds of the dynamics reveal a complex behaviour with an interesting feature, which does not appear in devices based on type-I materials, and hence is linked to the unique carrier capture/escape processes possible in type-II structures. C) 2015 AIP Publishing LLC. [http://dx.doi.org/10.1063/1.4906106]
\end{abstract}

Photonic materials based on type-II (staggered) band alignment such as $\mathrm{GaSb} / \mathrm{GaAs}$ quantum dots (QDs) have proved promising in applications such as charge-based memories $^{1}$ and solar cells. ${ }^{2}$ Many-particle interactions play a very important role in the dynamics of semiconductor nanostructures exhibiting such a band alignment. ${ }^{3}$ In these structures, spatial separation between unbound electrons and confined holes leads to greatly modified emission properties when compared with their type-I counterparts. Previous research on type-II GaSb/GaAs QDs utilizing time-resolved photoluminescence spectroscopy revealed a strong blue-shift of the emission energy accompanied by a significant reduction of the radiative lifetime at elevated carrier densities. ${ }^{4}$ However, a detailed analysis of the room temperature ultrafast carrier dynamics of these structures, which is essential for the improvement of the device performance, is still missing. Previously, two-colour pump-probe spectroscopy has proven to be very useful in revealing the carrier relaxation and escape processes of epitaxial and colloidal type-I QD structures. ${ }^{5,6}$ In this paper, we apply this technique to type-II based semiconductor optical amplifiers (SOAs) for the first time in both forward and reverse bias $(\mathrm{RB})$ mode and discover an interesting ultrafast feature that seems to be unique to type-II structures.

The structure comprises five layers of GaSb QDs grown on n-type GaAs substrate by molecular beam epitaxy. These QDs were formed by depositing 2.5 monolayers (MLs) of GaSb with a growth rate of $0.12 \mathrm{ML} \mathrm{s}^{-1}$, followed by $25 \mathrm{~nm}$ thick GaAs spacer. This multi-layered structure is surrounded by $1.25 \mu \mathrm{m} \mathrm{Al}_{0.3} \mathrm{Ga}_{0.7} \mathrm{As}$ barriers and finally covered with $100 \mathrm{~nm}$ of GaAs. A piece of wafer was processed into $1 \mathrm{~mm}$ long SOA with $4 \mu \mathrm{m}$ wide angled ridge. In order to provide good thermal conductivity, the SOA was then attached to a copper heat sink in a manner that allows light coupling to/ from both facets.

Fig. 1 shows the amplified spontaneous emission (ASE) peak energy and a ratio of the total emission intensity to applied current (termed the efficiency) as functions of increasing bias (in blue and red, respectively). Both quantities were examined over a wide range of currents exceeding 3 orders of magnitude and resulted in a total observed blueshift of $200 \mathrm{meV}$. It is clear from the graph that there are two regimes of behaviour. For low values of injection current $(<10 \mathrm{~mA})$, the ASE exhibits a large blue-shift and the efficiency increases. For larger currents $(>10 \mathrm{~mA})$, there is a reduction of both the ASE efficiency and the rate of change of ASE peak energy per unit current.

It has been commonly accepted that the giant blue-shift of the emission energy that occurs with increasing excitation is a fingerprint of staggered band alignment. ${ }^{7}$ The reasons attributed to this behaviour are threefold and occur simultaneously after injection of the carriers: band bending, ${ }^{8}$ capacitive charging, ${ }^{3,9}$ and state filling. ${ }^{3,4}$ In addition, the increase in efficiency for low currents can be understood in terms of

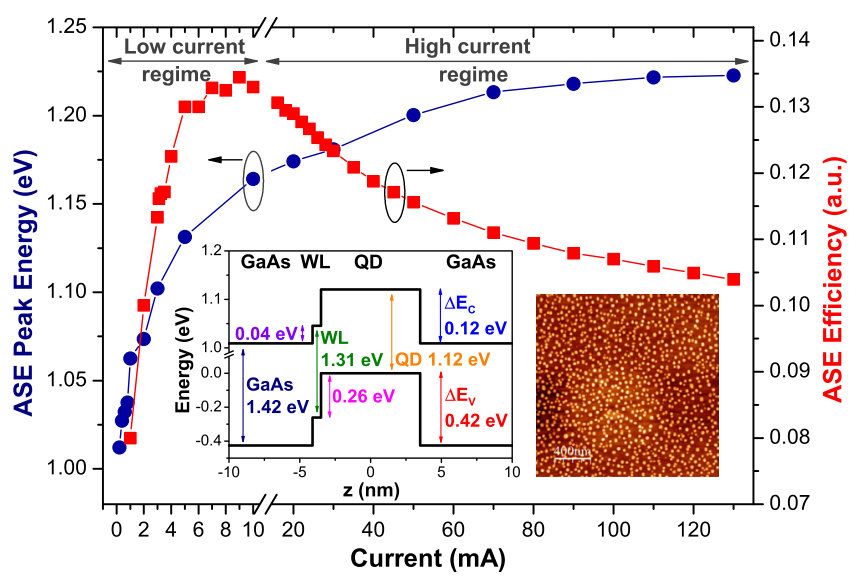

FIG. 1. Energy shift of ASE (blue curve) and ratio of ASE intensity to current (red curve) as a function of increasing current. Inset: calculated band alignment and AFM picture of examined QD structure. 
an increased overlap between electron and hole wavefunctions which result in a peak efficiency at $10 \mathrm{~mA} .{ }^{10}$

For higher currents, the reduction in blue-shift can be attributed to the lower confinement of higher energy hole wavefunctions which result in a lower charge density and hence a smaller contribution to the Coulomb potential, ${ }^{4,11}$ while the reduction in ASE efficiency can be attributed to the lower transfer rate of holes to the QD due to increased repulsion. ${ }^{12}$ Some research groups have associated the complete saturation of blueshift to the possible formation of a confining potential for electrons in the QD due to band bending effects and therefore type-I transitions at high carrier densities. ${ }^{11,13}$ In order to gain better insight into the band structure of our sample, we employed an eight band $\mathrm{k} \cdot \mathrm{p}$ formalism to calculate the band alignment and potentials for both carrier species. The dimensions of the QD $(32 \mathrm{~nm} \times 32 \mathrm{~nm}$ base size and $6.5 \mathrm{~nm}$ height) were obtained from atomic force microscopy (AFM) measurements and both a truncated pyramid shape and 24\% of antimony content were assumed. The result of the calculation is presented in the inset of Fig. 1. The valence band offset and conduction band discontinuity were found to be $425 \mathrm{meV}$ and $120 \mathrm{meV}$, respectively.

The dynamical properties of the SOA were examined at room temperature using two-colour differential pump-probe spectroscopy. ${ }^{5}$ Briefly, in this technique, femtosecond pulses of different wavelengths were used to pump and probe various transitions in the structure. In order to ensure independent wavelength tunability of the pump and probe pulses, the beam from a Ti:Sa laser was split into two parts; one part was used to pump an Optical Parametric Oscillator (OPO, $75 \%$ ) and the other to pump a highly nonlinear Photonic Crystal Fibre (PCF, 25\%). Thus, the OPO was the source of a tunable probe beam $(<300 \mathrm{fs}$, bandwidth $20 \mathrm{~nm})$, whereas optical filtering of the spectrally broadened PCF output provided the pump beam $(<200 \mathrm{fs}$, bandwidth $50 \mathrm{~nm})$. After applying a suitable delay between the beams and propagating through the SOA, low and high frequency lock-in amplifiers measured the relative $(\Delta R / R)$ change of the probe intensity due to pump-induced gain change. The wavelength of the pump pulses was set to $950 \mathrm{~nm}$ providing enough energy $(1.31 \mathrm{eV})$ to excite both wetting layer (WL) and QD e-h pairs, while the wavelength of probe pulses was varied from $1000 \mathrm{~nm}$ to $1100 \mathrm{~nm}(1.24 \mathrm{eV}-1.12 \mathrm{eV})$, which corresponds to transitions between electrons in either the dot, WL, or GaAs and hole levels in the dot (inset of Fig. 2).

In Fig. 2, we show the normalized gain dynamics at $0 \mathrm{~mA}$ and various wavelengths for the same pumping conditions. After arrival of the pump pulse to the structure (at $0 \mathrm{ps}$ delay), we observe initial absorption bleaching followed by a single exponential wavelength-dependent decay. This decay time is attributed to radiative recombination and varies from $970 \mathrm{ps}$ down to $300 \mathrm{ps}$ as the current increases from $0 \mathrm{~mA}$ to $120 \mathrm{~mA}$ (when probing at $1060 \mathrm{~nm}$ ). While our values are somewhat lower than expected for type-II structures, ${ }^{10}$ we note that in our case, the wavelengths correspond to the higher energy side of the spectrum, which results in shorter timescales. The reduction of the decay time with current can be understood in terms of an increased overlap of electron and hole wavefunctions at moderate current densities and hence faster recombination processes. ${ }^{14}$

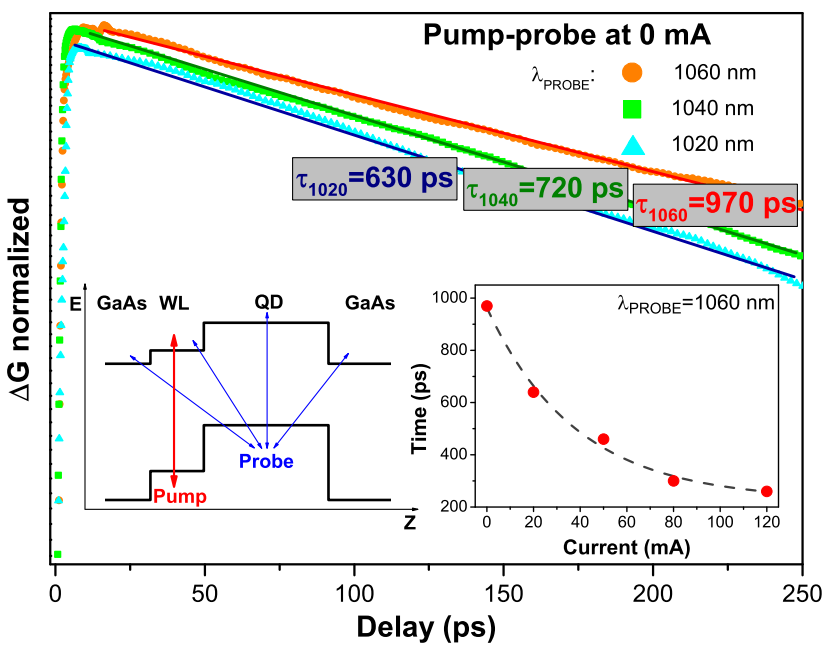

FIG. 2. Normalized change of the gain at different probe wavelengths at $0 \mathrm{~mA}$ bias. Inset: schematic of states pumped and probed during a single measurement and corresponding decay constant as a function of the bias current.

A more detailed examination of the initial few ps of the dynamics reveals an interesting feature, as shown in Fig. 3. Two dips are visible as the transmission increases due to the injection of carriers at higher energy. The first dip (at $0 \mathrm{ps)}$ coincides with the arrival of the pump pulse and can be attributed to weak multiphoton absorption when pump and probe pulses overlap. A similar dip occurs in the type-I, twocolour case ${ }^{5}$ and is a much weaker version of the strong coherent effects that are observed in all single colour pumpprobe measurements. The second dip occurs after $\sim 1 \mathrm{ps}$ and does not occur in similar measurements of type-I devices and so we attribute it to the type-II nature of the structure. As can be seen in Fig. 3, the properties of this dip do not show a clear dependence on bias current.

In contrast, Fig. 4 shows a strong dependence of the strength of the second dip on the pump power (number of injected carriers), while the first dip displays much less pump power dependence and thereby illustrates the difference in the underlying processes leading to each. Based on these observations, the appearance of a second dip can be understood in the following stages: 1 . Following the first dip

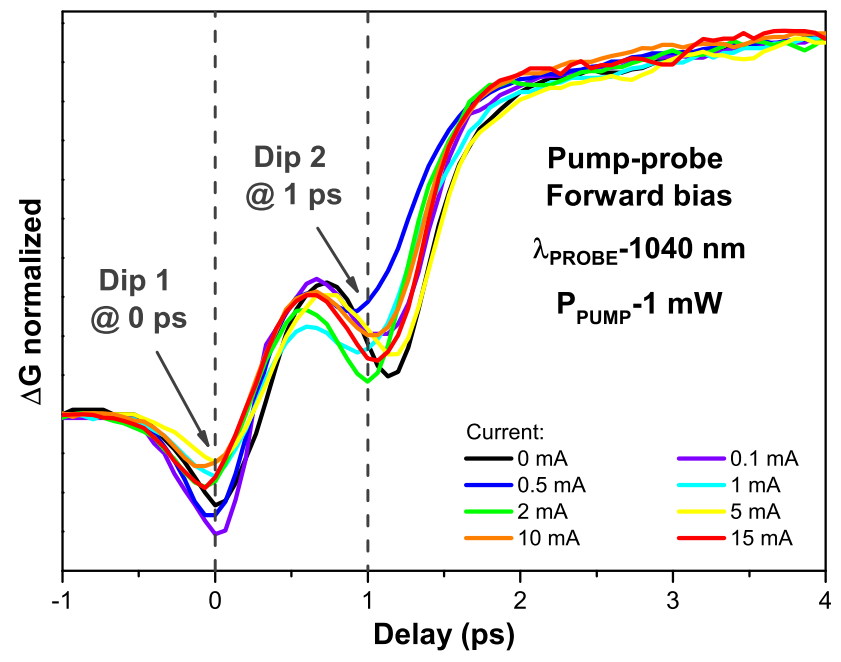

FIG. 3. Normalized first few ps of gain dynamics at various bias currents. Note the presence of dips at approximately $0 \mathrm{ps}$ and $1 \mathrm{ps}$. 


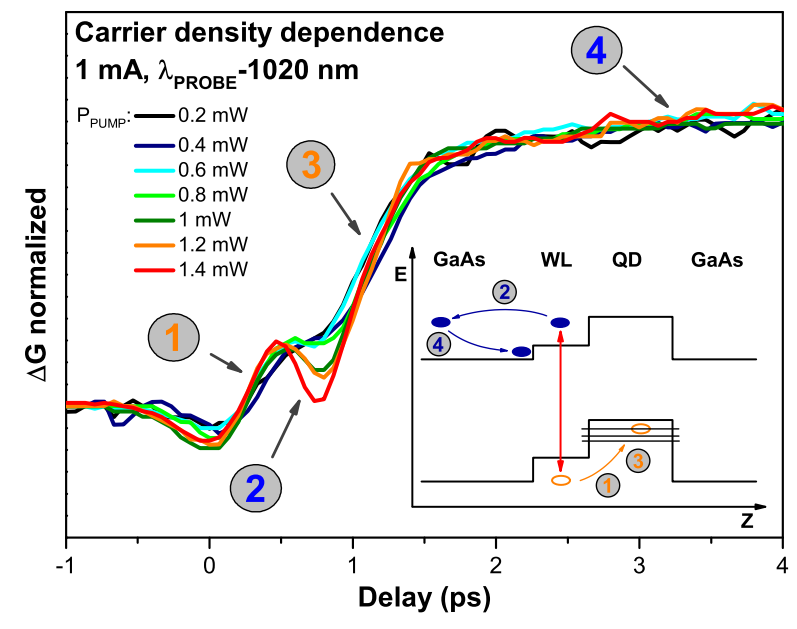

FIG. 4. Normalized first few ps of gain dynamics for increasing pump power level. Inset: schematic of various carrier transport/relaxation processes corresponding to gain changes labeled on main graph.

(which coincides with generation of electrons/holes in the $\mathrm{WL}$ ), the probe gain increases due to relaxation of WL electrons/holes into the GaAs/QD, respectively. 2. After $\sim 1 \mathrm{ps,}$ the unconfined electrons start to escape and diffuse away into the GaAs and the gain reduces (second dip). 3. The gain then increases due to further relaxation of holes into the dot. 4. After $\sim 1.5 \mathrm{ps}$, the final stage of the dynamics occurs with a much slower rise which we attribute to the Coulomb attraction of GaAs electrons to the vicinity of the dot by confined holes. Stages 1 and 3 are both predominantly related to hole capture dynamics and thus have similar slopes.

To further investigate this feature, the dynamics were recorded under RB in Fig. 5(a) with the recovery times presented in the inset. The reduction of the recovery time as the $\mathrm{RB}$ increases is similar to that measured for type-I QDs and suggests a thermalisation process for carrier relaxation. ${ }^{15}$ In the top figure, both the strength of the dip and the strength of the stage 4 (not clearly visible due to short time scale) decrease as the RB voltage increases. Thus, we conclude that as the $\mathrm{RB}$ increases, the injected unconfined electrons are quickly swept out and do not contribute to the subsequent dynamics. It is important to note that in reverse bias, confinement of electrons at the QD-GaAs interface due to band bending becomes possible and will lead to type-I style transitions ${ }^{8}$ and thereby to similar dynamics as seen at increased RB. The effect of changing the probe wavelength is shown in Fig. 5(b) and allows us to somewhat distinguish these type-I/type-II transitions. A much stronger dip can be seen at lower energies (red curve), while type-I style dynamics occur for higher energies (blue curve). This behaviour can be understood if we apply a reverse bias to the band-edge diagram in Fig. 2, as shown in inset of Fig. 5(b). In this situation, QD holes can recombine via either type-II or type-I channels with the former dominating at lower energies, while the latter dominates at higher energies.

In summary, we have presented a study of the ultrafast carrier dynamics in type-II GaAs/GaSb QDs. Off-peak measurements using a two-colour pump-probe technique demonstrate a strong dependency of carrier escape processes on the applied reverse bias voltage, the probing wavelength, and the number of injected carriers. The first few picoseconds of the
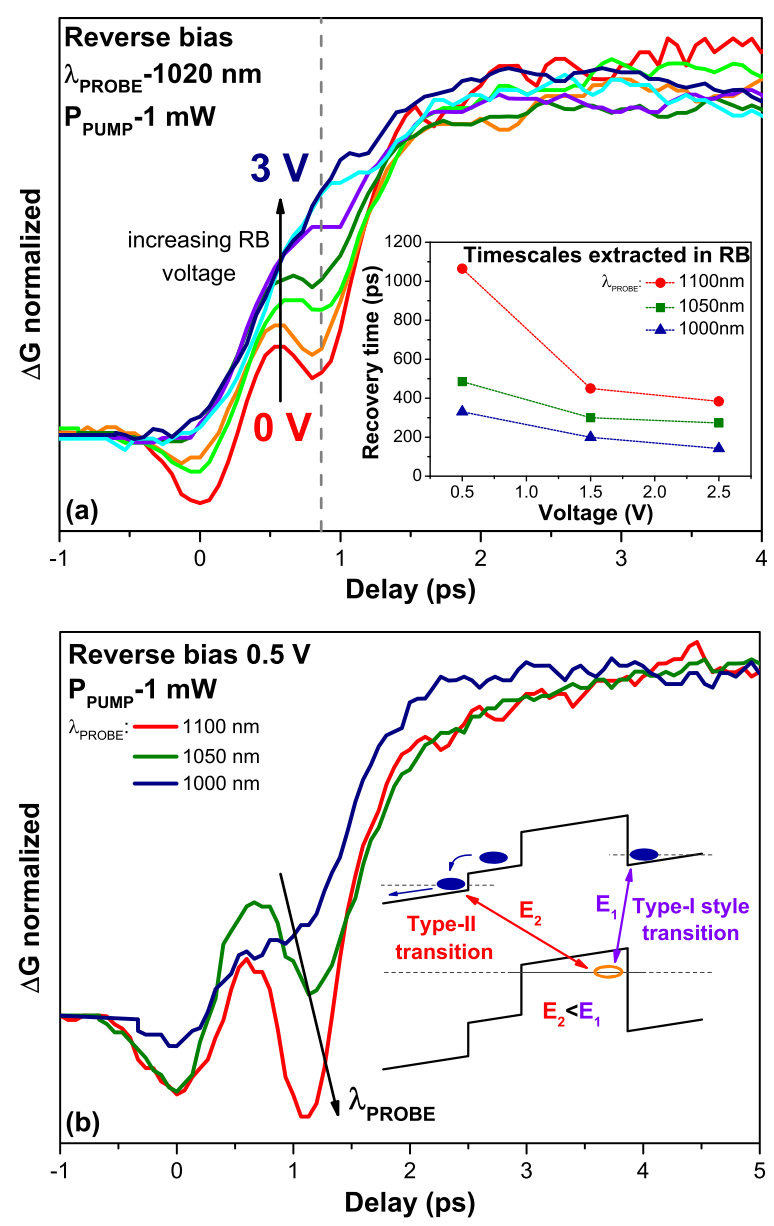

FIG. 5. (a) Normalized first few ps of gain dynamics in RB regime as RB voltage increases. Inset in (a) shows gain recovery timescales as a function of the probe wavelength/RB voltage. (b) Effect of changing probe wavelength in $\mathrm{RB}$ with schematic of possible shorter and longer wavelength processes. The longer wavelength process $\left(E_{2}\right)$ involves unconfined electrons and is thus termed type-II, while the shorter wavelength one $\left(E_{1}\right)$ involves confined electrons and is termed type-I.

dynamics reveal an interesting feature, which does not appear in devices based on type-I materials and is linked to the unique carrier processes possible in type-II structures. The onset of type-I transitions can also be seen for increasing $\mathrm{RB}$ voltages. In addition, the extracted decay times in forward and reverse bias may provide important information from an application point of view.

The authors would like to thank Dr. Liam Lewis for fabrication of SOAs and Dr. Bryan Kelleher for proof reading the manuscript. This work was conducted under the framework of the Irish Government's Programme for Research in Third Level Institutions Cycle 5, National Development Plan 2007-2013 with the assistance of the European Regional Development Fund. The authors acknowledge the support from the University of California Lab Fees Research Program (Grant No. 12-LR-238568).

\footnotetext{
${ }^{1}$ M. Hayne, R. Young, E. Smakman, T. Nowozin, P. Hodgson, J. Garleff, P. Rambabu, P. Koenraad, A. Marent, L. Bonato et al., J. Phys. D: Appl. Phys. 46, 264001 (2013).

${ }^{2}$ J. Hwang, A. Martin, J. Millunchick, and J. Phillips, J. Appl. Phys. 111, 074514 (2012).
} 
${ }^{3}$ L. Müller-Kirsch, R. Heitz, A. Schliwa, O. Stier, D. Bimberg, H. Kirmse, and W. Neumann, Appl. Phys. Lett. 78, 1418 (2001).

${ }^{4}$ K. Gradkowski, T. Ochalski, N. Pavarelli, H. Liu, J. Tatebayashi, D. Williams, D. Mowbray, G. Huyet, and D. Huffaker, Phys. Rev. B 85, 035432 (2012).

${ }^{5}$ I. O'Driscoll, T. Piwonski, C. Schleussner, J. Houlihan, G. Huyet, and R. Manning, Appl. Phys. Lett. 91, 071111 (2007).

${ }^{6}$ P. Kambhampati, Acc. Chem. Res. 44, 1 (2010).

${ }^{7}$ F. Hatami, N. Ledentsov, M. Grundmann, J. Böhrer, F. Heinrichsdorff, M. Beer, D. Bimberg, S. Ruvimov, P. Werner, U. Gösele et al., Appl. Phys. Lett. 67, 656 (1995).

${ }^{8}$ D. Alonso-Álvarez, B. Alén, J. M. García, and J. M. Ripalda, Appl. Phys. Lett. 91, 263103 (2007).

${ }^{9}$ P. Hodgson, R. Young, M. A. Kamarudin, P. Carrington, A. Krier, Q. Zhuang, E. Smakman, P. Koenraad, and M. Hayne, J. Appl. Phys. 114, 073519 (2013).
${ }^{10}$ F. Hatami, M. Grundmann, N. Ledentsov, F. Heinrichsdorff, R. Heitz, J. Böhrer, D. Bimberg, S. Ruvimov, P. Werner, V. Ustinov et al., Phys. Rev. B 57, 4635 (1998).

${ }^{11}$ J. Tatebayashi, A. Khoshakhlagh, S. Huang, G. Balakrishnan, L. Dawson, D. Huffaker, D. Bussian, H. Htoon, and V. Klimov, Appl. Phys. Lett. 90, 261115 (2007).

${ }^{12}$ C.-C. Tseng, W.-H. Lin, S.-Y. Wu, S.-H. Chen, and S.-Y. Lin, J. Cryst. Growth 323, 466 (2011).

${ }^{13}$ S. Morozov, D. Kryzhkov, A. Yablonsky, A. Antonov, D. Kuritsin, D. Gaponova, Y. G. Sadofyev, N. Samal, V. Gavrilenko, and Z. Krasilnik, J. Appl. Phys. 113, 163107 (2013).

${ }^{14}$ K. Gradkowski, N. Pavarelli, T. Ochalski, D. Williams, J. Tatebayashi, G. Huyet, E. O'Reilly, and D. Huffaker, Appl. Phys. Lett. 95, 061102 (2009).

${ }^{15}$ T. Piwonski, J. Pulka, G. Madden, G. Huyet, J. Houlihan, E. A. Viktorov, T. Erneux, and P. Mandel, Appl. Phys. Lett. 94, 123504 (2009). 